\title{
3D-printed patient individualised models vs cadaveric models in an undergraduate oral and maxillofacial surgery curriculum: Comparison of student's perceptions
}

\author{
Lukas B. Seifert $^{1}$ (D) | Benedikt Schnurr ${ }^{1}$ | Carlos Herrera-Vizcaino ${ }^{1}$ | Amira Begic ${ }^{2}$ | \\ Florian Thieringer $^{3}$ | Frank Schwarz ${ }^{2}$ | Robert Sader ${ }^{1}$
}

${ }^{1}$ Department of Oral, Cranio-Maxillofacial, and Facial Plastic Surgery, University Hospital Frankfurt, Goethe University, Frankfurt, Germany

${ }^{2}$ Polyclinic for Dental Surgery and Implantology, Carolinum Dentistry University Institute $\mathrm{gGmbH}$ of the Goethe University Frankfurt am Main, Frankfurt am Main, Germany

${ }^{3}$ Department of Oral, Cranio-Maxillofacial Surgery, University Hospital, Basel University, Basel, Switzerland

\section{Correspondence}

Lukas B. Seifert, Department of Oral, Cranio-Maxillofacial, and Facial Plastic Surgery, University Hospital Frankfurt, Theodor-Stern-Kai 7, 60590 Frankfurt, Germany.

Email: lukasbenedikt.seifert@kgu.de

\begin{abstract}
Background: Recent advances in 3D printing technology have enabled the emergence of new educational and clinical tools for medical professionals. This study provides an exemplary description of the fabrication of 3D-printed individualised patient models and assesses their educational value compared to cadaveric models in oral and maxillofacial surgery.

Methods: A single-stage, controlled cohort study was conducted within the context of a curricular course. A patient's CT scan was segmented into a stereolithographic model and then printed using a fused filament 3D printer. These individualised patient models were implemented and compared against cadaveric models in a curricular oral surgery hands-on course. Students evaluated both models using a validated questionnaire. Additionally, a cost analysis for both models was carried out. P-values were calculated using the Mann-Whitney $U$ test.

Results: Thirty-eight fourth-year dental students participated in the study. Overall, significant differences between the two models were found in the student assessment. Whilst the cadaveric models achieved better results in the haptic feedback of the soft tissue, the 3D-printed individualised patient models were regarded significantly more realistic with regard to the anatomical correctness, the degree of freedom of movement and the operative simulation. At $3.46 €$ (compared to $6.51 €$ ), the 3D-printed patient individualised models were exceptionally cost-efficient.

Conclusions: 3D-printed patient individualised models presented a realistic alternative to cadaveric models in the undergraduate training of operational skills in oral and maxillofacial surgery. Whilst the 3D-printed individualised patient models received positive feedback from students, some aspects of the model leave room for improvement.
\end{abstract}

KEYWORDS

3D printing, 3D rapid prototyping, dentoalveolar surgery, oral and maxillofacial surgery, simulation training 


\section{1 | INTRODUCTION}

One of the biggest challenges in both medical and dental training is to provide students with the skills needed for their future work. In daily practice, a wide range of psychosocial to practical-technical skills must be mastered at a very high level, especially if they involve irreversible operations. ${ }^{1}$

For the training of irreversible operations, simulation training is commonly used in medical and dental education ${ }^{2}$ with various examples in the fields of cardiovascular resuscitation, ${ }^{3}$ emergency medicine $^{4}$ and surgical training. ${ }^{3,5}$ One of its main advantages compared to traditional chair or bedside teaching is the possibility to practice a certain skill as often as necessary until a high level of competence is achieved without jeopardising patients' health ("the patient died, but we can try again"). ${ }^{6}$ However, the use of modern high-fidelity simulators often also involves high acquisition costs which limits their accessibility for medical teachers.

For the training of surgical procedures in particular, cadaveric models are frequently used as an alternative to high-fidelity simulators. ${ }^{7,8}$ However, the accessibility to human cadaveric models for surgical training is limited due to ethical and also financial restrictions, and animal cadaveric models do not always provide the anatomical precision needed to train a certain surgical procedure.

Recent advances in 3D printing technology have enabled the emergence of new educational and clinical tools for medical professionals. Since 2009 , various attempts have been made to produce non-cadaveric educational models for otorhinolaryngology, ${ }^{9,10}$ neurosurgery ${ }^{11-13}$ and oral and maxillofacial surgery. ${ }^{14-16}$ The development of the fused deposition modelling technique (FDM) especially has enabled clinicians to produce individualised patient models with the help of inexpensive consumer market 3D printers at very low costs. In addition, today a variety of free software solutions are available that allow a standardised and easy workflow from a medical imaging dataset to the production of a stereolithographic model.

Despite these recent technological advancements, little is known about the actual educational benefits of 3D-printed individualised patient models compared to other training devices. The majority of the present literature focuses on the technical realisation rather than the curricular implementation of 3D-printed individualised patient models. Some studies have evaluated their educational use in undergraduate education; for example, Kröger et al fabricated individualised training models for veneer preparation, dental bonding and caries excavation in a dental hands-on course. Their results showed 3D printing to be a viable method, and the models received a positive feedback from students with a wide range of possibilities for improvement. ${ }^{17}$ A further study by Marty et al compared students perceptions of 3D-printed individualised patient models vs commercial available models in a conservative dentistry course and found significant advantages in the 3D-printed model. ${ }^{18}$

To our knowledge, there are no previous studies reporting on the educational value of 3D-printed individualised patient models compared to porcine cadaveric animal models (pig mandible) in the acquisition of operative skills within an undergraduate oral and maxillofacial surgery curriculum. Therefore, the present study aims to provide an exemplary description of the fabrication and implementation of 3D-printed individualised patient models in an oral and maxillofacial surgery hands-on course for fourth-year dental students. From this goal, two research questions emerged: Was there any difference between the cadaveric and the 3D-printed model in terms of educational value; and how cost-efficient were the 3D-printed models compared to the cadaveric models?

\section{2 | MATERIALS AND METHODS}

\section{1 | Design and printing of the 3D individualised patient model}

A CT scan was obtained from a young patient (age 18, male) needing oral surgery (extraction of displaced/impacted third molars). All the data of the computed tomography were anonymised and were converted into stereolithographic (.stl) files using the free Slicer ${ }^{\circledR}$ software (version 4.10.1. for Mac, https://commonfund.nih.gov/ bioinformatics). Further modifications on the 3D model were made using the publicly available Meshmixer ${ }^{\circledR}$ software (Figure 1A). The mandibular dental arch was isolated, and the teeth 20-21, 23-26 and 28-29 were removed to enable students to practice the dissection of a mucoperiosteal flap for sealing the alveolar socket. To simulate the realistic extraction of the impacted third molars, a periodontal ligament space separating the teeth and the bone was set to a space of $1 \mathrm{~mm}$. The simulated impacted tooth was affixed in alveolar bone by support structure plus silicon. Furthermore, the pulp chambers of teeth 19 and 30 were slightly enlarged to practice the resection and retrograde filling of root tips. Finally, the model was fused with the.stl file of a Frasaco ${ }^{\circledR}$ base plate to allow the mounting of the 3D-printed individualised model in our patient simulators (KaVo Dental $\mathrm{GmbH}$ ). The processed.stl file was then exported in the MakerBot Desktop Software version 3.9.1 and printed with a MakerBot Replicator 2nd Generation 3D Printer (MakerBot Industries) in biodegradable white polylactic acid filament (PLA+, Esun Industrial Co., Ltd.) with the following settings on the printer: $0.2 \mathrm{~mm}$ resolution, $20 \%$ infill, rafts "on" and supports "on." PLA was extruded at $215^{\circ} \mathrm{C}$ onto the build platform. To simulate oral mucosa, silicone rubber (Wagnersil 22 NF, Wagner Dental GmbH und Co. KG) was applied via disposable tongue blades and $10-\mathrm{ml}$ syringes (Figure $1 \mathrm{~B}$ ) to the finished models. The final models (Figure $1 \mathrm{C}$ ) were mounted in patient simulators (KaVo Dental GmbH) using a knurled nut (DIN 466, www.gedexshop.de) (Figure 1D).

\subsection{Cadaveric animal models}

The cadaveric animal models that were used for this study were porcine mandibles. These mandibles were provided by a local 
FIGURE 1 Design, fabrication and curricular implementation of the $3 \mathrm{D}$ individualised patient model. A, Modified 3D model of a patient's mandible with displaced/impacted third molars. The teeth 20-21, 23-26 and 28-29 were isolated and digitally removed. $\mathrm{B}$, Application of silicone rubber to simulate the oral gingiva and mucosa. C, Final models printed with silicone "oral mucosa." D, Mounted models in a patient simulator (KaVo Dental GmbH, Biberach, Germany). E, Students were working in groups of two of whom one practised on the cadaveric model, whilst the other practised on the 3D-printed individualised patient model and vice versa [Colour figure can be viewed at wileyonlinelibrary. com]
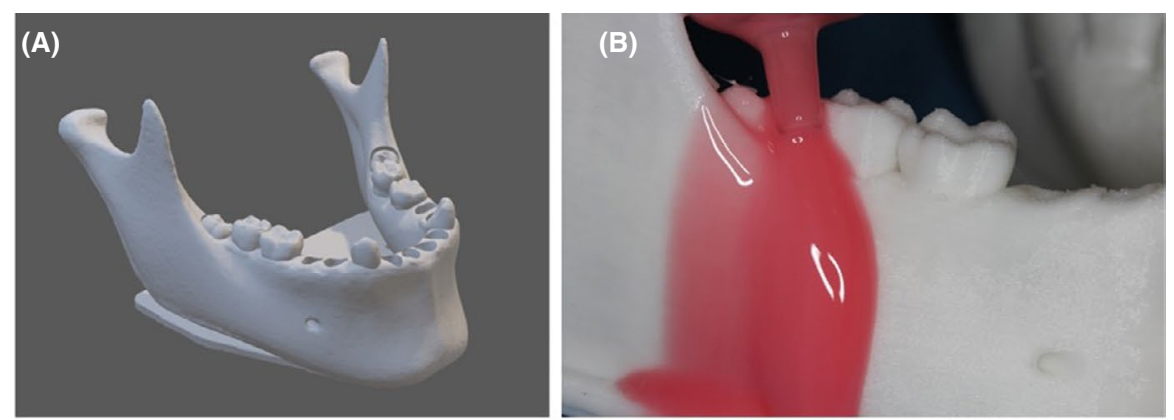

(C)
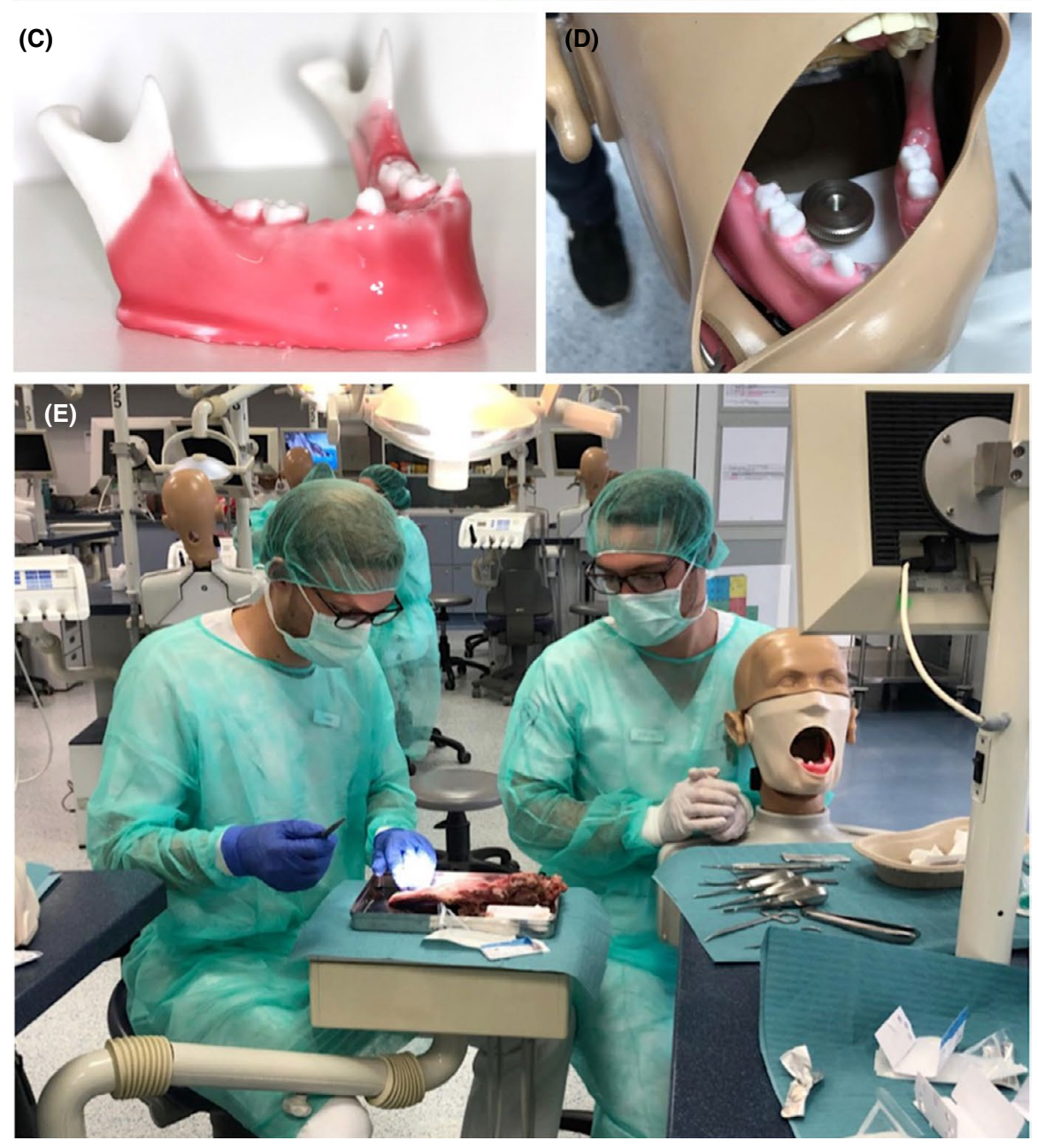

butcher and split into half before the intervention; thus, every pair of students could practice on the right or left side of one porcine mandible.

\section{3 | Study participants}

Study participants were undergraduate dental students at Goethe University in Frankfurt/Main, Germany at the end of their fourth year of a 5-year programme completing their obligatory surgical training. Their surgical training began in the first clinical semester (6th semester out of 10) with regular interactive lectures in oral and maxillofacial and general surgery which included live demonstrations on real patients. Furthermore, they had to participate in practical hands-on courses to practice the placement of local anaesthesia (7th semester) and the extraction of teeth (8th semester). Before participating in this study's intervention, students already had passed two clinical semester treating real patients in conservative dentistry and dental prosthetics, had extracted at least eight single- and multirooted teeth and had to regularly assist in oral surgeries and were regarded as experienced.

Participation in the study was voluntary and took place after written informed consent, which was revocable at any time. Basic data regarding student age, sex and duration of study were collected using a questionnaire. The study was conducted according to ethical principles of the Helsinki Declaration (Ethical Principles for Medical 
Research Involving Human Subjects), and the local ethics committee stated that no further approval was necessary.

\section{4 | Intervention}

The study was carried out at the end of the 8th semester of an obligatory oral surgery hands-on course for dental students. The learning objectives of this course were as follows: the dissection of a mucoperiosteal flap, the osteotomy of impacted third molars, the dissection of a free mucosal graft and the resection of a root tip.

The course took place in Goethe University's dental simulation laboratory. Two experienced oral surgeons were filmed performing each of the above operations on a pig's mandible and on 3D-printed individualised patient model explaining every step whilst being observed by the students on monitors. Afterwards, students practised every operation on cadaveric models and on 3D-printed individualised patient models which were mounted in phantom heads. Students worked in groups of two: one practised on the cadaveric model, whilst the other practised on the 3D-printed individualised patient model and vice versa (Figure 1E). Whilst practicing, students were observed by the oral surgeons and corrected if necessary. In total, the course lasted for 240 minutes.

\subsection{Evaluation of the models}

At the end of each procedure, students were asked to fill out a questionnaire, which was a translation and adaptation into German of the one previously used and published by Nickel et al ${ }^{19}$ A native English speaker translated the questionnaire into German. One question was modified into four sub-questions about the usefulness of the simulation for every learning objective. In this questionnaire, each statement could be rated on a Likert scale ranging from 1 = "does not apply at all" to 10 = "fully applies."

\section{6 | Cost analysis}

A cost analysis was performed to estimate the manufacturing costs of the 3D-printed individualised patient models compared to the cadaveric models. For this, the material costs per model and the working time of a student assistant for the production of the 3D-printed individualised patient models were included.

\section{7 | Data analysis}

Microsoft Excel 2016 for Mac (version 15.24) and GraphPad Prism (version 6.0 for Mac, GraphPad Software) were used to conduct the statistical evaluation and to apply the Mann-Whitney $U$ test to test the significance of the ordinally distributed data.

\section{3 | RESULTS}

\section{1 | Study participation}

Thirty-eight of 39 students decided to participate in the study and filled out a questionnaire. Of those 38 questionnaires, 34 were included in the analysis. Four questionnaires had to be excluded because they were incomplete. Twenty-six study participants were female, whilst 12 were male which corresponds to the normal gender distribution at Goethe University's dental school. The curricular implementation of the 3D-printed patient individualised models within the oral surgery hands-on course for dental students was completely feasible in terms of time management and students' perception.

\section{2 | Model evaluation}

Overall, significant differences between the two models were found in the student assessment (Figure 2). The cadaveric models achieved slightly better scores in the general evaluation of the reality of the simulation compared to the 3D-printed patient individualised models. In particular, the haptic feedback of the gingiva and mucosa of the cadaveric models was evaluated significantly more realistic than the silicone mucosa in the 3D-printed patient individualised models. In the free-text commentaries (Table 1), the silicone mucosa was often described as being too fragile. This was also reflected in the assessment of tasks that require manipulation of the soft tissue like intraoral suturing or the dissection of a mucoperiosteal flap. However, the 3D-printed individualised patient models achieved significantly better scores regarding the anatomical correctness of the simulation. The 3D-printed patient individualised models were also rated significantly higher in haptic feedback and as a useful simulation for learning about intraoral incisions. The clearest difference was found in the evaluation of the degree of freedom of movement of the simulation. Here, 3D-printed patient individualised models were rated significantly more realistic than the cadaveric models. Students also assessed the operating room OR simulation as significantly more realistic in the 3D-printed patient individualised models compared to the cadaveric models. In the free-text commentaries, students particularly praised the realistic human anatomy and the possibility to mount the 3D-printed patient individualised models in a phantom head to achieve a more realistic simulation of performing intraoral operations. A less fragile silicone gingiva and a better differentiation between the colour of teeth and bone were the most desired suggested improvements to the 3D-printed patient individualised models (Table 1).

\section{3 | Cost analysis}

The production costs for twenty 3D-printed individualised patient models amounted to 69.2 Euro, including material (21 Euro for $1 \mathrm{~kg}$ of PLA Filament; 35.90 Euro for $1 \mathrm{~L}$ of silicone rubber) and personnel 

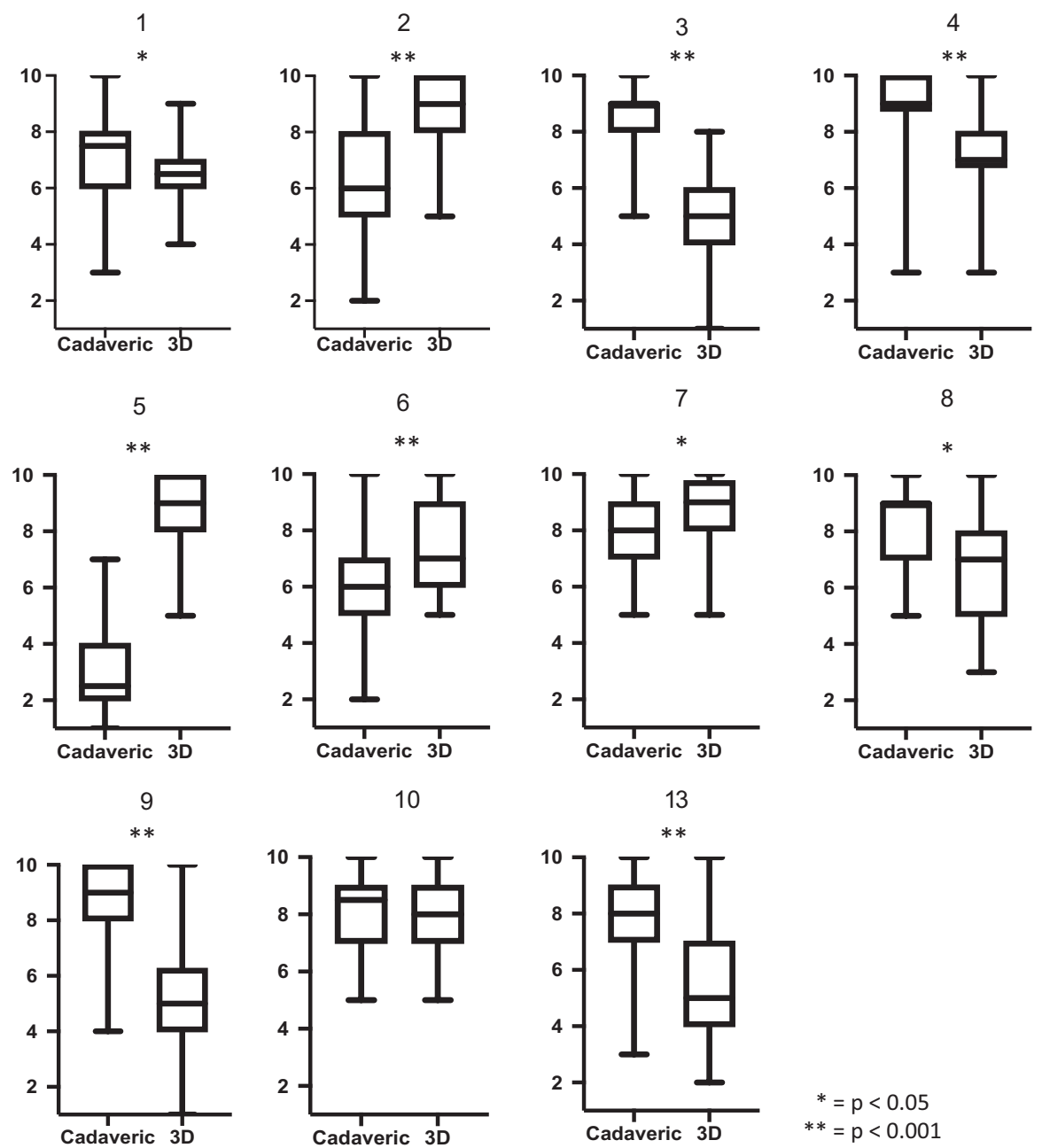

10

13
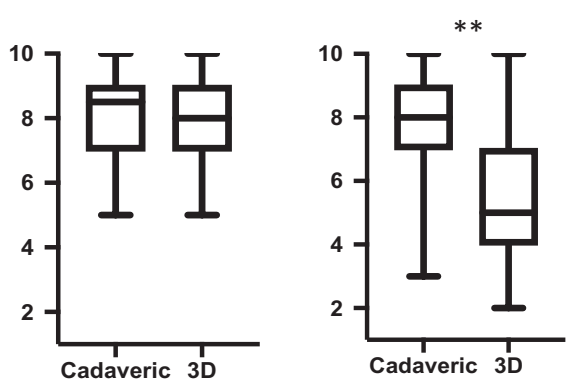

$\begin{aligned} * & =p<0.05 \\ * * & =p<0.001\end{aligned}$

FIGURE 2 Results of the students' assessment of the 3D-printed individualised patient models compared to the cadaveric models. (1) The simulator resembles reality. $(n=38$; range $3 D=5$; range cadaveric $=7$; median $3 D=6.5$; median cadaveric $=7.5 ; P=.02)$. $(2)$ The anatomical assignment of each part is correct. $(n=38$; range $3 D=5$; range cadaveric $=8$; median $3 D=9 ;$ median cadaveric $=6 ; P=<.001)$.

(3) The haptic feedback of soft tissue is realistic. $(n=38$; range $3 D=7$; range cadaveric $=5$; median $3 D=5$; median cadaveric $=9$; $P=<.001$ ).

(4) The haptic feedback of hard tissue is realistic. $(n=38$; range $3 D=7$; range cadaveric $=7$; median $3 D=7$; median cadaveric $=9 ; P=<.001$ ).

(5) The degree of freedom of movement with the instruments is realistic. $(n=38$; range $3 D=5$; range cadaveric $=6$, median $3 D=9$; median cadaveric $=2.5 ; P=<.001)$. (6) The OR simulation resembles reality. $(n=38$; range $3 D=5 ;$ range cadaveric $=8$, median $3 D=7 ;$ median cadaveric $=6 ; P=<.02)$. (7) The simulator is a useful instrument to learn intraoral incisions. $(n=38$; range $3 D=5 ;$ range cadaveric $=5$, median $3 \mathrm{D}=9$; median cadaveric $=8 ; P=<.001$ ). (8) The simulator is a useful instrument to learn intraoral suturing. ( $\mathrm{n}=38 ;$ range $3 \mathrm{D}=7$; range cadaveric $=5$, median $3 \mathrm{D}=7$; median cadaveric $=9 ; P=<.007)$. (9) The simulator is a useful instrument to learn the dissection of mucoperiosteal flaps. ( $n=38$; range $3 D=9$; range cadaveric $=6$, median $3 D=5$; median cadaveric $=9 ; P=<.001$ ). (10) The simulator is a useful instrument to learn intraoral osteotomies. $(n=38$; range $3 D=5$; range cadaveric $=5$, median $3 D=8$; median cadaveric $=8.5$; $P=<.35)$. (11) The simulator is a useful instrument to learn the resection of a root tip. $(n=38$; range $3 D=8$; range cadaveric $=7$; median $3 \mathrm{D}=5 ;$ median cadaveric $=8 ; P=<.001)$

costs (student assistant, 14 Euro per hour for 3.5 hours). The costs for 20 cadaveric models amounted to 130.90 Euro, including the professional disposal of the models and taxes (Table 2).

\section{4 | DISCUSSION}

The present study aimed to provide an exemplary description of the fabrication and curricular implementation of 3D-printed individualised patient models in an oral and maxillofacial surgery hands-on course for fourth-year dental students. Moreover, we investigated for the first time if there was any difference in educational value between 3D-printed individualised patient models and cadaveric models from a student's perspective and how cost-effective each type of simulation model was.

Overall, significant differences between 3D-printed individualised patient models and cadaveric models were found. In particular, the cadaveric model was assessed significantly more realistic in terms of soft tissue simulation and achieved better scores in tasks in which soft tissue manipulation plays an integral part (ie the 
TABLE 1 Student evaluation of the 3D-printed individualised patient models

I liked on the model, that...

Anatomical situation corresponds to real patient

Mounting in a phantom head was possible, and the bone was realistic

Represents anatomical situation well, and intraoral work represents realistic suturing, etc

One could practice suture techniques intraorally

Mask reflects intraoral situation, osteotomy at 3D model good with low rotation speed, real size reproduction at 3D model

Simulation intraoral, human anatomy

Mounting in phantom head was possible, human anatomy

Intraoral work, training incision lines for osteotomy of third molars, impaction of the third molars

It was possible to practice the techniques in the phantom head and practice the handling of the instruments intraorally

Root and tooth anatomy ratios were much more realistic than in the cadaveric models, in the phantom head practice much more realistic than in the cadaveric models

It was as realistic as possible in terms of freedom of motion. Practicing the osteotomy of third molars was very good.

The patient simulation was given (by the installation of the jaw in the head). It was great fun!

It was also possible to mount the 3D jaw into the phantom head, and it has also been relatively realistic in terms of bone simulation. Besides, it is more hygienic!

Visually, the 3D jaw is very real, excellent integration of modern 3D technology (3D printer, CT data set) from research for teaching

It was in the phantom head (freedom of movement), and it has considered human anatomy

You can mount it in the phantom head, good handling

You could mount it into the phantom head patient simulation

Anatomical design ++, very close to reality (one is restricted in his movements, as well as with his view)

Number of teeth and shape identical in humans

Anatomy is correct, mountable in phantom head

There was a second 3D model in human design that could also be mounted into the phantom head

It is closest to the patient situation from the optical view

It was very close to reality, visually and because of the patient's positioning

I think it would be better, if.

- Gingiva was more stable, and there was less tearing.

- The soft tissue would be more realistic, and the tooth colour and bone could be separated better visually.

- Gingiva material needs to be improved for realistic presentation.

- The gingiva would not tear so quickly.

- Simulation of the gingiva more realistic, easier distinction of teeth and bones in resection of the root tip.

- The teeth would differ in colour from the bone.

- The silicone of the gingiva would be a bit more stable.

- Root tips could be improved; teeth could be pulled out of the model; soft tissue would have a higher tear strength, and presence of periosteum, we can practice on real human heads, for example from the anatomy.

- To improve the silicone for the suture techniques. In comparison, it tears too fast.

- The soft tissue would be of another material because it is very easily torn.

- Material of the gingiva would be more realistic (very brittle) rips and is unrealistic in handling, and the plastic material would be much harder and more brittle.

- The gingiva would be a bit more stable and better attached to the plastic.

- The material would be harder (gingiva), and one could do the complete osteotomy of third molars.

- The gingiva would be more stabel, and one could extract the whole tooth in the osteotomy.

- Other material for the soft tissue (should not tear quickly), other colour of tooth and bone to better differentiate it.

- Root tips would differentiate more clearly from the bone.

- It would be possible to differentiate bones better from tooth to osteotomy.

- It would be possible to use another material for soft tissue simulation that does not tear down so quickly.

dissection of a mucoperiosteal flap). On the other hand, 3D-printed individualised patient models were perceived as significantly more realistic in terms of anatomical correctness and the OR simulation by students. The possibility to mount the 3D-printed individualised patient models in a phantom head and hence simulate intraoral operations with a limited degree of freedom of movement with the instruments was perceived as their biggest strength compared to the cadaveric models. Even though it was not directly reported in the evaluation, for socio-cultural reasons some groups may prefer to not use porcine material which is often used in oral surgery training. In this case, 3D-printed models provide a possible alternative.
Previous studies have also sought to investigate the educational value of 3D-printed individualised patient models in dental education before. Kröger et al fabricated three individualised training models using intraoral scans for a hand-on course in operative dentistry. Experienced volunteer students performed a caries excavation and composite filling on one of the training models. Overall, these models received positive feedback and students liked the idea of using patient individualised training models. ${ }^{17}$ However, in the particular study, there was no control group that performed the caries excavation and composite filling on a gold-standard model, that is extracted teeth, which limits its findings regarding the reality 
TABLE 2 Comparison of costs between 3D-printed individualised patient models and cadaveric models

\begin{tabular}{llll} 
Cost type & 3D model & & Cadaveric model \\
\hline Material costs & PLA 42 g & $0.88 €$ & $3.57 €$ \\
& Silicone $7 \mathrm{~mL}$ & $0.25 €$ & \\
\hline Personnel costs & $2.33 €$ & & None \\
\hline Disposal costs & none & $59.5 €$ \\
\hline Cost per model & $3.46 €$ & $6.54 €$ \\
\hline $\begin{array}{c}\text { Total costs (for } \\
20 \text { models) }\end{array}$ & $69.2 €$ & $130.9 €$ \\
\hline
\end{tabular}

of simulation. Another study by Marty et al compared students' perceptions of 3D-printed individualised patient models to their views on Frasaco ${ }^{\circledR}$ industrial models in an operative dentistry course and came to similar conclusions. ${ }^{18}$ Students evaluated the 3D-printed individualised patient models significantly better in terms of simulation reality and simulation of caries. However, their study was conducted in a non-curricular voluntary setting and study participants might have been biased because they were accustomed to the Frasaco ${ }^{\circledR}$ models since their second year of study. Other than these studies, we could show that 3D-printed individualised patient models also present an alternative to cadaveric models in the acquisition of operational skills in oral and maxillofacial surgery within an undergraduate curriculum and that these models can be fabricated relatively easily at a low price. The cost of manufacturing a single 3D-printed individualised patient model came to $3.46 €$, which was significantly lower than the cost of a cadaveric model (6.54€). The cost of medical education has far-reaching implications regarding its impartation and policymaking. ${ }^{20}$ Frenk et al reported a worldwide spending on medical education of about 100 billion with a rising tendency, which is becoming a real challenge on health system performance. ${ }^{21,22}$ In this sense, 3D-printed models could be a more cost-effective strategy to improve medical education. Of course, it has to be mentioned that these results cannot be generalised. In low- and middle-income countries that have less access to 3D printing technology, the use of cadaveric models may still be cheaper.

Despite positive feedback from the students, our 3D-printed individualised patient models left room for improvement in terms of soft tissue simulation. With ongoing technological progress in computer-assisted design and computer-assisted manufacturing, it is only a question of time until the fabrication of more realistic soft tissue simulations will be possible at an affordable price. The use of 3D printing for the fabrication of simulation devices is in the earliest stages and improvements are likely as the technology develops.

We believe that these technical limitations do not outweigh the advantages provided by 3D-printed individualised patient models. The possibility to fabricate simulators on the basis of radiological data of a real patient allows students to get a better understanding of the problems of certain pathologies, such as impacted and displaced third molars, and comprehend the complexity of an individual patient anatomy.
Of course, there are limitations to this study. First of all, with 38 participating students, the sample size of our study was rather small which limits the statistical power of its findings. Furthermore, only two types of models were compared. There are commercially available simulation models which might provide a more realistic training scenario but were not used due to their high acquisition costs. Last, the use of materials to fabricate the models was limited to PLA filament and silicone rubber which leaves the question if other materials that might provide a more realistic simulation. This should be addressed in future studies.

\section{CONCLUSION}

We described a method to fabricate low-cost patient individualised models based on real patient situations using 3D printing technology. Overall, these models presented a realistic alternative to cadaveric models in the training of operational skills from the spectrum of oral and maxillofacial surgery within an undergraduate curriculum. Whilst the 3D-printed individualised patient models received positive feedback from students in a hands-on course, some aspects of the model leave room for improvement.

\section{ORCID}

Lukas B. Seifert iD https://orcid.org/0000-0002-0069-9961

\section{REFERENCES}

1. Sweet J, Wilson J, Pugsley L. Chairside teaching and the perceptions of dental teachers in the UK. Br Dent J. 2008;205(10):565-569.

2. Buchanan JA. Use of simulation technology in dental education. J Dent Ed. 2001;65(11):1225-1231. http://www.jdentaled.org/conte nt/jde/65/11/1225.full.pdf. Accessed August 23, 2019.

3. Aqel AA, Ahmad MM. High-fidelity simulation effects on CPR knowledge, skills, acquisition, and retention in nursing students. Worldviews Evidence-Based Nurs. 2014;11(6):394-400.

4. Small SD, Wuerz RC, Simon R, Shapiro N, Conn A, Setnik G. Demonstration of high-fidelity simulation team training for emergency medicine. Acad Emerg Med. 1999;6(4):312-323.

5. Sutherland LM, Middleton PF, Anthony A, et al. Surgical simulation: a systematic review. Ann Surg. 2006;243(3):291-300.

6. Aggarwal R, Mytton OT, Derbrew M, et al. Training and simulation for patient safety. Qual Saf Heal Care. 2010;19(Suppl 2):i34-i43.

7. Gilbody J, Prasthofer A, Ho K, Costa M. The use and effectiveness of cadaveric workshops in higher surgical training: a systematic review. Ann R Coll Surg Engl. 2011;93(5):347-352.

8. Henn RF, Shah N, Warner JJP, Gomoll AH. Shoulder arthroscopy simulator training improves shoulder arthroscopy performance in a cadaveric model. Arthrosc J Arthrosc Relat Surg. 2013;29(6):982-985.

9. Chan HHL, Siewerdsen JH, Vescan A, Daly MJ, Prisman E, Irish JC. 3D rapid prototyping for otolaryngology-head and neck surgery: applications in image-guidance, surgical simulation and patient-specific modeling. Warburton D, ed. PLoS One. 2015;10(9):e0136370.

10. Mowry SE, Jammal H, Myer C, Solares CA, Weinberger P. A novel temporal bone simulation model using $3 \mathrm{D}$ printing techniques. Otol Neurotol. 2015;36(9):1562-1565.

11. Ploch CC, Mansi CSSA, Jayamohan J, Kuhl E. Using 3D printing to create personalized brain models for neurosurgical training and preoperative planning. World Neurosurg. 2016;90:668-674. 
12. Ryan JR, Almefty KK, Nakaji P, Frakes DH. Cerebral aneurysm clipping surgery simulation using patient-specific 3D printing and silicone casting. World Neurosurg. 2016;88:175-181.

13. Wen G, Cong Z, Liu K, et al. A practical 3D printed simulator for endoscopic endonasal transsphenoidal surgery to improve basic operational skills. Child's Nerv Syst. 2016;32(6):1109-1116.

14. Lioufas PA, Quayle MR, Leong JC, McMenamin PG. 3D printed models of cleft palate pathology for surgical education. Plast Reconstr surgery Glob open. 2016;4(9):e1029.

15. Lambrecht JTH, Berndt D, Christensen AM, Zehnder M. Haptic model fabrication for undergraduate and postgraduate teaching. Int J Oral Maxillofac Surg. 2010;39(12):1226-1229.

16. Werz SM, Zeichner SJ, Berg B-I, Zeilhofer H-F, Thieringer F. 3D printed surgical simulation models as educational tool by maxillofacial surgeons. Eur J Dent Educ. 2018;22(3):e500-e505.

17. Kröger E, Dekiff M, Dirksen D. 3D printed simulation models based on real patient situations for hands-on practice. Eur J Dent Educ. 2017;21(4):e119-e125.

18. Marty M, Broutin A, Vergnes J-N, Vaysse F. Comparison of student's perceptions between 3D printed models versus series models in paediatric dentistry hands-on session. Eur J Dent Educ. 2019;23(1):68-72.
19. Nickel F, Kowalewski K-F, Rehberger F, et al. Face validity of the pulsatile organ perfusion trainer for laparoscopic cholecystectomy. Surg Endosc. 2017;31(2):714-722.

20. Tsang MC. Cost analysis for improved educational policymaking and evaluation. Educ Eval Policy Anal. 1997;19(4):318-324.

21. Walsh $\mathrm{K}$. Cost and value in medical education - What we can learn from the past? J R Coll Physicians Edinb. 2014;44(4):324-327.

22. Frenk J, Chen L, Bhutta ZA, et al. Interprofessionelles Lernen in Tutorien für Lernende in Ergo- und Physiotherapie, Medizin und Pfle. Lancet. 2010;376:1923-1958.

How to cite this article: Seifert LB, Schnurr B, Herrera-

Vizcaino C, et al. 3D-printed patient individualised models vs cadaveric models in an undergraduate oral and maxillofacial surgery curriculum: Comparison of student's perceptions. Eur J Dent Educ. 2020;24:799-806. https://doi.org/10.1111/ eje.12522 